MiGRANAL

(dihydroergotamine mesylate nasal spray)

$4 \mathrm{mg} / \mathrm{mL}$ Nasal Spray

THERAPEUTIC CLASSIFICATION: Migraine Therapy

PHARMACOLOGICAL CLASSIFICATION: 5-HT, Receptor Agonist

ACTIONS AND CLINICAL PHARMACOLOGY: Dihydroergotamine displays agonist activity at the 5- $\mathrm{HT}_{10}$ receptor, which, by reducing $5-\mathrm{HT}$ neuronal function and/or contracting elements of the cranial vasculature and/or suppressing neurogenic inflammation, is believed to underlie its anti-migraine efficacy. It also displays affinity for the 5-HTic receptor and antagonistic activity at the 5- $\mathrm{HT}_{2}$ subtype. Dihydroergotamine displays blocking actions at alpha adrenoreceptors, with a direct stimulating effect on the smooth muscle of peripheral blood vessels. Its tonic effect on capacitance vessels (veins) is particularly pronounced, compared to its effects on resistance vessels (arterioles). Dihydroergotamine differs from ergotamine by being more potent with respect to its adrenergic blocking actions and less potent with respect to its capacity to produce arterial vasoconstriction, but it maintains a marked venoconstrictor effect. Dihydroergotamine reduces the incidence and degree of nausea, photophobia, and phonophobia. Intranasally administered dihydroergotamine is rapidly absorbed in a dose-independent manner ( $\mathrm{t}_{\max }=$ approximately 45 minutes). Significant relief of migraine begins within approximately 30 minutes following administration of MIGRANAL (dihydroergotamine mesylate nasal spray). Once pain is relieved, the incidence of return of pain within 24 hours is low. The bioavailability of dihydroergotamine administered intranasally is $43 \%$. Dihydroergotamine is $93 \%$ bound to plasma proteins and has a steady-state volume of distribution of about $800 \mathrm{~L}$. The parent drug constitutes 70 to $80 \%$ of plasma concentrations of drug-related materials. The nasal spray form of dihydroergotamine, like most parenteral dose routes, is not subject to first-pass hepatic metabolism. The total body clearance is about $1.5 \mathrm{~L} / \mathrm{min}$. refiecting mainly a hepatic clearance. Plasma elimination of dihydroergotamine is biphasic with a mean terminal half-life of 10 hours. The major route of excretion is via the bile in the feces. After intranasal administration, the urinary recovery of parent drug amounts to about $2 \%$ of the dose.

INDICATIONS AND CLINICAL USE: MIGRANAL (dihydroergotamine mesylate nasal spray) is indicated for the treatment of migraine headaches, with or without aura, in adults. MIGRANAL is not indicated for prophylactic therapy or for the management of hemiplegic or basilar migraine.

CONTRAINDICATIONS: MIGRANAL (dihydroergotamine mesylate nasal spray) is contraindicated in patients who have previously shown hypersensitivity to ergot alkaloids, or to any of the components of MIGRANAL. MIGRANAL is contraindicated in patients having conditions predisposing to vasospastic reactions such as known peripheral arterial disease, coronary heart disease (in particular unstable or vasospastic angina), septic conditions and shock, vascular surgery, obliterative vascular disease, inadequately controlled hypertension, and severely impaired hepatic function. Dihydroergotamine possesses oxytocic properties and, therefore, should not be administered during pregnancy. It is likely that dihydroergotamine is excreted in breast milk. MIGRANAL is therefore contraindicated for nursing mothers.

WARNINGS: Dihydroergotamine could cause vasospastic reactions, including angina, although it seems to do so less frequently than ergotamine. This action appears to be dose-related. These reactions are manifested by intense arterial vasoconstriction, producing signs and symptoms of peripheral vascular ischemia (e.g., muscle pains, numbness, coldness and pallor or cyanosis of the digits), angin or unusual syndromes, such as mesenteric ischemia. Consequently, MIGRANAL (dihydroergotamine mesylate nasal spray) should be discontinued immediately if signs or symptoms of vasoconstriction develop. The solution used in MIGRANAL was especially developed for intranasal administration and must not be injected.

PRECAUTIONS: Pediatric Use: Safety and effectiveness of MIGRANAL (dihydroergotamine mesylate nasal spray) in children have not been established. Use in Elderly: Experience with the use of MIGRANAL in patients aged over 65 years is limited. Orug Inferactions: The concomitant use of ora contraceptives by female patients does not appear to influence the disposition of MIGRANAL (dihydroergotamine mesylate nasal spray). MIGRANAL should not be used with vasoconstrictors because the combination may cause a further elevation of blood pressure. Concurrent use of vasoconstrictor agents including ergotamine, sumatriptan and nicotine may enhance the risk of vasoconstriction. Twenty four hours should elapse before taking sumatriptan following administration of MIGRANAL. This will avoid additive vasospastic effects. Conversely, MIGRANAL can be taken six hours following the administration of sumatriptan. Although there have been reports that propranolol may potentiate the vasoconstrictive action of ergotamine by synergism upon B-blockade, the results of a limited clinical study ( $\mathrm{n}=8$ ) did not indicate a safety problem associated with the administration of MIGRANAL in subjects already receiving propranolol. The concomitant use of macrolide antibiotics such as erythromycin. troleandomycin or josamycin with MIGRANAL should be avoided since these antibiotics may increase the plasma level of dihydroergotamine. Nursing Mothers: It is likely that dihydroergotamine is excreted in human milk, although it is not known at which concentration, while it is known that ergotamine is excreted in breast milk and may cause vomiting, diarrhea, weak pulse and unstable blood pressure in breast-fed infants. 8ecause of the potential for these serious adverse events in breast-fed infants, nursing mothers should not use MIGRANAL (dihydroergotamine mesylate nasal spray). Information for the Palient: Currently available data have not demonstrated drug abuse and psychological dependence with MIGRANAL (dihydroergotamine mesylate nasal spray). However due to the chronicity of migraines, patients should be advised not to exceed recommended dosages. Patients should be advised to report immediately to the physician any of the following: numbness or tingling in the fingers and toes, muscle pain in the arms and legs, weakness in the legs, pain in the chest, temporary speeding or slowing of the heart rate, swelling, or itching. Patients should be advised of the importance of priming the applicator (pump 4 times) prior to administration to ensure correct dosage. No more than four sprays $(2.0 \mathrm{mg}$ ) of MIGRANAL should be administered for any single migraine headache attack. No more than eight sprays $(4.0 \mathrm{mg})$ of MIGRANAL should be administered during any 24 hour period. The maximum weekly dosage is 24 sprays $(12.0 \mathrm{mg}$ ) of MIGRANAL (see DOSAGE AND ADMINISTRATION).

ADVERSE REACTIONS: The most commonly reported adverse events associated with the use of MIGRANAL (dihydroergotamine mesylate nasal spray) in placebo-controlled, double-blind studies for the treatment of migraine headaches, and not reported at an equivalent incidence by placebo-treated patients, were rhinitis, nausea, taste disturbance and application site reaction. In clinical trials these events were transient and self-limiting, and generally did not result in patient drop-out. The following table lists the adverse events experienced at incidences greater than $1 \%$.

Adverse Events Reported in Double-Blind Placebo-Controlled Studies for the Treatment of Migraine Headaches (Reported at Incidences $\geq 1 \%$ )

\begin{tabular}{lll}
$\begin{array}{l}\text { Adverse Reactions } \\
\text { According to Body System }\end{array}$ & $\begin{array}{l}\text { Rate of Occurrence (\%) } \\
\text { MIGRANAL (N=642) }\end{array}$ & $\begin{array}{l}\text { Rate ol Occurrence (\%) } \\
\text { Placebo ( } N=632)\end{array}$ \\
\hline
\end{tabular}

\section{Central Nervous System}

Dizziness

Somnotence

Fatigue

Confusion

Nervousnes

Asthenia

Gastrointestinal System

Taste disturbance
Adverse Reactions

According to Body System

Rate of Occurrence (\%) MIGRANAL ( $N=642$ )

Rate of Occurrence (\%) Placebo $(\mathrm{N}=632)$

Gastrointestinal System (cont'd.)

Vomiting

Diarrhea

Dysphagia

Respiratory System

Rhinitis'

Application site reaction

Pharyngitis

Nasal discharge

Sinusitis

Musculo-skeletal System

Myalgia

Stiffness

Autonomic Nervous System

Hot flushes

Sweating increased

Dry mouth

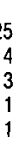

Rhinitis includes reports of nasal/nose congestion, nose dryness, nose edema, rhinitis, rhinorrhea and excessive sneezing.

In a few patients who have taken oral dihydroergotamine continuously over years, development of fibrotic changes, in particular of the pleura and the retroperitoneum, has been observed. Chest tightness/pain was seen in earlier studies although the incidence was less than $1 \%$ and a causal relationship was not established. In rare cases, vascular spasms may occur, particularly in the lower extremities. If signs of vascular spasms are observed, MIGRANAL should be discontinued and treatment with a peripheral vasodilator initiated (see SYMPTOMS AND TREATMENT OF OVERDOSAGE). SYMPTOMS AND TREATMENT OF OVERDOSAGE: There have been no reports of acute overdosage with MIGRANAL (dihydroergotamine mesylate nasal spray). The symptoms of an acute oral dihydroergotamine overdose are similar to those of an ergotamine overdose, although there is less

pronounced nausea and vomiting with dihydroergotamine. These symptoms include the following: peripheral signs and symptoms of vasospasm (e.g. numbness, tingling, pain and cyanosis of the extremities associated with diminished or absent peripheral pulses); respiratory depression; an increase and/or decrease in blood pressure usually in that order; confusion, delirium, convulsions and coma; and/or some degree of nausea, vomiting and abdominal pain. The treatment of an overdosage is symptomatic under close monitoring of the cardiovascular and respiratory systems. Treatment includes discontinuation of the drug, local application of warmth to the affected area and nursing care to prevent tissue damage; in case of severe vasospasms, vasodilators should be administered (e.g. sodium nitroprusside, phentolamine or dihydralazine). In the case of coronary constriction, appropriate treatment such as nitroglycerin should be initiated.

DOSAGE AND ADMINISTRATION: Prior to administration of MIGRANAL (dihydroergotamine mesylate nasal spray) the nasal spray applicator must be primed (pumped 4 times). In order to let the drug be absorbed through the skin in the nose, patients should not inhale deeply through the nose while spraying or immediately after spraying. For best results, treatment should be initiated at the first symptom or sign of an attack. However, MIGRANAL can be used at any stage of a migraine attack. At the first sign or symptoms of a migraine headache, or as early as possible after the onset of headache pain, one spray $(0.5 \mathrm{mg}$ ) of MIGRANAL (dihydroergotamine mesylate nasal spray) should be administered into each nostril. If the condition has not sufficiently improved approximately fifteen minutes later, an additional spray $(0.5 \mathrm{mg})$ of MIGRANAL should be administered to each nostril. The usual dosage required to obtain optimal efficacy is a total dosage of four sprays $(2.0 \mathrm{mg})$ of MIGRANAL. MIGRANAL is exclusively indicated for the symptomatic treatment of migraine attacks. MIGRANAL should not be used as a prophylactic therapy. Significant relief of migraine begins within approximately 30 minutes following nasal administration of MIGRANAL. No more than four sprays $(2.0 \mathrm{mg})$ should be administered for any single migraine attack. An interval of at least 6-8 hours should be observed before treating another migraine attack with MIGRANAL or any drug containing dihydroergotamine or ergotamine. No more than eight sprays $(4.0 \mathrm{mg})$ (corresponding to the use of 2 ampoules) should be administered during any 24 hour period. The maximum weekly dosage is 24 sprays $(12.0 \mathrm{mg}$ ) of MIGRANAL. MIGRANAL does not need to be administered with an antiemetic, as is recommended with the parenteral form of dihydroergotamine mesylate, since the administration of the nasal spray form is not associated with nausea and vomiting to the same extent as the parenteral form. Once pain is relieved, the incidence of pain return within 24 hours is low. Once the nasal spray applicator has been prepared, it must be discarded with any remaining drug atter $B$ hours. Spray applicator has been prepared,

Trade Name: MIGRANAL; Common Name: 9-10-Dihydro-12'-hydroxy-2'-methyl-

$5^{\prime} \alpha$ (phenylmethyl) ergotaman -3', 6', 18-trione monomethane sulfonate Structural Formula:

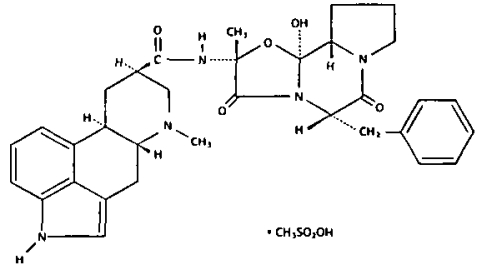

Molecular Formula: $\mathrm{C}_{33} \mathrm{H}_{3} \mathrm{~N}_{5} \mathrm{O}_{5} \cdot \mathrm{CH}_{4} \mathrm{SO}_{3} ;$ Molecular Weight: $679.8 ;$ Description: White or oft-white fine, crystalline, hygroscopic powder. Moderately soluble in water. $p K_{a}$ in ethanol-water (1:1): $6.35 \pm$ 0.05 . pH in solution: $4.4-5.4$. Dihydroergotamine mesylate melts with strong decomposition between $220^{\circ} \mathrm{C}$ and $240^{\circ} \mathrm{C}$. Composition of MIGRANAL: Each ampoule of MIGRANAL contains $4.0 \mathrm{mg}$ dihydroergotamine mesylate USP as well as the following non-medicinal ingredients: anhydrous caffeine, carbon dioxide, anhydrous dextrose, and water. Storage Requirements: MIGRANAL should be stored at room temperature $\left(15^{\circ} \mathrm{C}\right.$ to $\left.25^{\circ} \mathrm{C}\right)$

AVAILABILITY OF DOSAGE FORM: MIGRANAL (dihydroergotamine mesylate nasal spray) is available as a clear, colourless to faintly yellow solution, in $1 \mathrm{~mL}$ amber glass ampoules. MIGRANAL is provided as a package of three units, each unit consisting of one ampoule with a plastic breaker sleeve and a nasal spray applicator.

References:

1. Migranal Product Monograph 1996. Sandoz Canada Inc.

2. Gallagher RM, Ventura D, DiSerio FJ et al. Arch of Neurol 1996 (in press).

3. Ziegler D, Ford R, Kriegler J et al. Neurology 1994;44:447-453.

Product Monograph available on request. 